\title{
X-ray Absorption Spectroscopy of Liquid Methanol Microjets: Bulk Electronic Structure and Hydrogen Bonding Network
}

\author{
Kevin R. Wilson, ${ }^{\dagger, »}$ Matteo Cavalleri, ${ }^{\S}$ Bruce S. Rude," R. D. Schaller, $\$$ T. Catalano," \\ A. Nilsson, ${ }^{\S, \perp}$ R. J. Saykally, $*$, and L. G. M. Pettersson $*, \S$ \\ Department of Chemistry, University of California, Berkeley, California 94720, FYSIKUM, \\ Stockholm University, AlbaNova University Center, S-10691 Stockholm, Sweden, Advanced Light Source, \\ Lawrence Berkeley National Laboratory, Berkeley, California 94720, and Stanford Synchrotron Radiation \\ Laboratory, Menlo Park, California 94025
}

Received: February 17, 2004; In Final Form: September 22, 2004

\begin{abstract}
We have measured the X-ray absorption (XA) spectrum of liquid (298 K) methanol at the oxygen and carbon $\mathrm{K}$ edges. The $4 \mathrm{a}_{1}$ orbital at the $\mathrm{O} \mathrm{K}$ edge exhibits a pronounced sensitivity to the formation of intermolecular hydrogen bonds, with significant differences observed between the vapor and bulk spectra, whereas the $\mathrm{C} \mathrm{K}$ edge reveals only subtle corresponding spectral changes. Comparison with DFT computed spectra of model methanol clusters indicates that the bulk liquid comprises long chains $(n>6)$ and rings of hydrogen-bonded monomers.
\end{abstract}

\section{Introduction}

Our knowledge of the electronic and geometrical structure of liquids has advanced dramatically over the past decade due to rapid progress in both theory and experiment. However, the microscopic structure of hydrogen-bonded liquids remains a vigorously debated subject. Classical molecular dynamics simulations have offered some new microscopic insights, but developing realistic force fields that properly account for manybody effects in the bulk liquid continues to be a challenge. ${ }^{1} \mathrm{Ab}$ initio molecular dynamics simulations eliminate the need for predetermined force-fields and the method has become wellestablished as a simulation tool. Wilson et al., have recently reported a new X-ray spectroscopy technique for the study of bulk liquids and liquid-vapor interfaces. ${ }^{2-4}$ In the initial applications, we described the first measurement of intermolecular surface relaxation in liquids (water and methanol), ${ }^{4}$ as well as the presence of a previously unrecognized type of "acceptor only" species at the liquid water surface. ${ }^{3}$ Recently, these experimental findings have been corroborated by new ab initio molecular dynamics (CPMD) simulations reported by Kuo and Mundy, ${ }^{5}$ who find that $19 \%$ of surface molecules of $298 \mathrm{~K}$ water are "acceptor only". Here we study the bulk properties of liquid methanol by X-ray absorption spectroscopy, using liquid microjets to introduce the volatile samples into the high vacuum environment of a synchrotron endstation and as an effective means of avoiding sample photodamage.

Soft X-ray absorption spectroscopy (XAS) is a powerful structural tool commonly used to study the bonding of adsorbates to metal surfaces through the ability to determine local geometry and bond lengths. ${ }^{6}$ A main advantage of XAS is the

* Corresponding Authors. E-mail: R.J.S., saykally@uclink4.berkeley.edu; L.G.M.P., lgm@physto.se.

† Present address: Lawrence Berkeley National Laboratory, Chemical Sciences Division, Berkeley, CA 94720

$\doteqdot$ University of California.

$\S$ Stockholm University.

"Lawrence Berkeley National Laboratory.

${ }^{\perp}$ Stanford Synchrotron Radiation Laboratory. ability to selectively excite individual atoms within a molecule and thereby examine specific chemical bonds (e.g., $\mathrm{C}=\mathrm{O}$, $\mathrm{C}-\mathrm{C}, \mathrm{O}-\mathrm{H}$, etc.). Traditionally, near-edge X-ray absorption fine structure (NEXAFS) experiments have characterized the rehybridization of covalent bonds, which can yield information about which orbitals are involved in the formation of a surface chemical bond. ${ }^{7}$ Although NEXAFS is most sensitive to changes in intramolecular bond lengths and angles, there are a number of recent studies ${ }^{3,4,8-15}$ examining the changes in electronic structure of liquid water, ice and $\mathrm{H}_{2} \mathrm{O}$ clusters upon the rearrangement of intermolecular hydrogen bonding (HB). Together these studies indicate that the $\mathrm{O} K$ edge XAS is particularly sensitive to broken donor hydrogen bonds located, e.g., within liquid water, ${ }^{14,15}$ on the surface of ice, ${ }^{15,16}$ or at the liquid-gas interface. ${ }^{3}$ As expected, water in its various phases is a natural starting point to investigate the local changes in electronic structure induced by $\mathrm{HB}$. In this paper, we extend these studies of intermolecular hydrogen bonds to methanol, which might be considered the next logical step in a detailed examination of the changes in electronic structure that occur upon condensation within the bulk hydrogen-bonded liquid. The general strategy outlined here is to first obtain high-resolution XA spectra of molecular (gas phase) methanol whereupon spectral changes induced by condensation can be evaluated. Subsequently, density functional theory calculations, utilizing small methanol clusters, are used to further interpret the experimental XA spectra, yielding new insights into the HB network of the bulk liquid.

\section{X-ray Absorption of Liquids}

XAS entails the removal of a core electron and its promotion into an unoccupied molecular orbital or continuum state. The excitation process obeys dipole selection rules yielding spectral intensity from transitions between the $1 \mathrm{~s}$ ( $\mathrm{K}$ shell) orbital to excited states of mainly p symmetry. The core hole is strongly localized, allowing individual atoms within molecules to be independently excited. For methanol, the carbon $(\sim 289 \mathrm{eV})$ and oxygen $\mathrm{K}$ edge $(\sim 530 \mathrm{eV})$ spectral intensity will be sensitive 


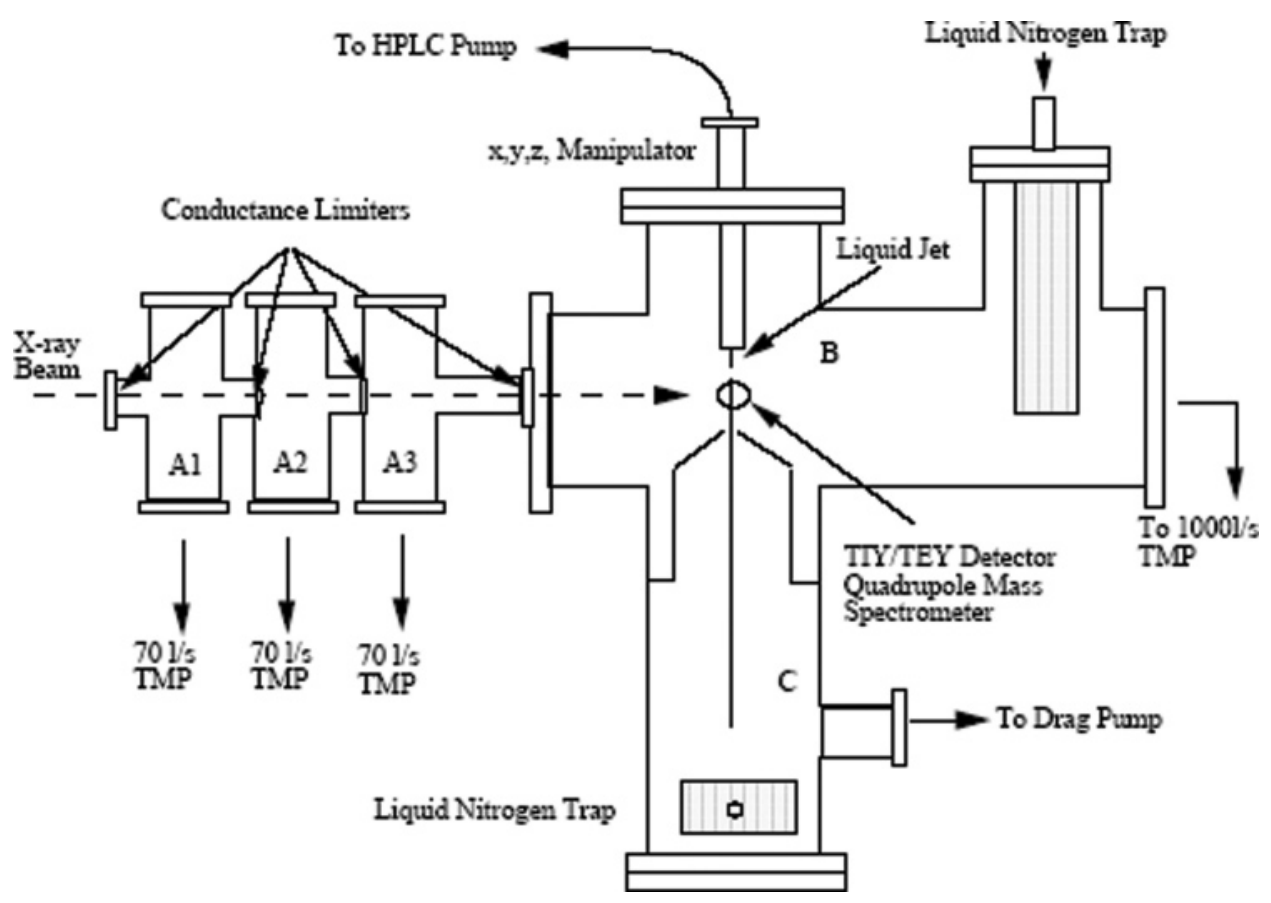

Figure 1. Schematic of the liquid microjet endstation. Section A consists of three differentially pumped stages. The pressure in A1 is near the beamline base pressure of $1 \times 10^{-9}$ Torr. Section B $\left(8 \times 10^{-5}\right.$ Torr $)$ houses the liquid jet that intersects the focused X-ray beam at $90^{\circ}$. Section $\mathrm{C}\left(1 \times 10^{-3}\right.$ Torr $)$ is $\sim 1 \mathrm{~m}$ in length and houses a liquid nitrogen trap upon which the liquid microjet is frozen. A more detailed description of the experiment can be found in ref 19 .

to either $\mathrm{C}$ or $\mathrm{O}$ local $\mathrm{p}$ contributions in the excited orbitals, respectively. In this way, changes in the molecular electronic structure (e.g., rehybridization) can be monitored upon condensation. Furthermore, alcohols are unique molecules, allowing one to investigate both the orbital changes occurring around the oxygen atom due to formation of an intermolecular hydrogen bond as well as the electronic effects of weaker intermolecular forces acting on the alkyl group.

The X-ray absorption coefficient can be probed directly, as in traditional transmission experiments, or indirectly, by detecting the emission of secondary electrons, ions, neutrals or fluorescent photons emitted in the core-hole decay process. For isolated molecules in the gas phase, spectra measured by monitoring the total ion (TIY) or electron (TEY) yield give similar results, when various ion fragmentation pathways are accounted for. However, in the condensed phase this is not the case due to the difference in escape depths of charged particles, photons and neutrals. ${ }^{10,17,18}$ For example, fluorescent photons have an escape depth of many microns, whereas secondary electrons, which dominate the TEY, are thought to be bulk sensitive determined by their average kinetic energy distribution $(<20 \mathrm{eV})$ and various other material properties. ${ }^{6}$ Ions, atoms or molecules desorb directly from the interface making this detection mode sensitive to only the outermost surface layer $(1-5 \AA) .{ }^{17}$ By measuring the gas and liquid-phase TEY as a function of X-ray excitation energy, XA spectra of isolated molecules can be compared with those embedded in a hydrogen bonding matrix. In this way we can isolate the effects of intermolecular hydrogen bonds on the XA spectra. Furthermore, microscopic insight into the electronic effects of hydrogen bond formation can be investigated using DFT calculations of the electronic structure of small methanol clusters.

\section{Experimental Design}

The experiments were conducted at beamline 8.0 of the Advanced Light Source, Lawrence Berkeley National Labora- tory. The small spot size and high photon flux of this undulator beamline are essential for obtaining a high quality spectrum of a $20 \mu \mathrm{m}$ diameter liquid methanol jet. The endstation used for soft X-ray studies of volatile liquids has been described in detail elsewhere. ${ }^{2,19}$ Briefly, the endstation consists of three sections, labeled A, B and C in Figure 1. The first section houses three stages of differential pumping with the last stage held near the base pressure of the $\mathrm{X}$-ray beamline $\left(\sim 1 \times 10^{-9}\right.$ Torr $)$. Within section $\mathrm{B}$, a $20 \mu \mathrm{m}$ diameter continuous stream of liquid methanol is injected into vacuum at a velocity of $\sim 20 \mathrm{~m} / \mathrm{s}$ with a mechanical backing pressure of $\sim 30$ atm generated by a commercial HPLC pump. The microjet then travels $1-2 \mathrm{~mm}$, where it is intersected at $90^{\circ}$ by the photon beam $(100 \times 200$ $\mu \mathrm{m})$, after which it is dumped through a $500 \mu \mathrm{m}$ aperture into a liquid nitrogen trap located $1 \mathrm{~m}$ from the nozzle. The base pressure during the normal operation of a $20 \mu \mathrm{m}$ diameter liquid methanol jet, maintained by a $1000 \mathrm{~L} / \mathrm{s}$ turbomolecular pump located behind a liquid nitrogen baffle, is $8 \times 10^{-5}$ Torr. Copper wires appropriately biased served as total yield detectors. Gas phase measurements were conducted by simply translating the liquid target $1-4 \mathrm{~cm}$ from the focused X-ray beam. Wilson et al. have shown ${ }^{19}$ that the liquid-vapor interface of water is generated under near thermal equilibrium conditions with this $20 \mu \mathrm{m}$ jet arrangement (i.e., the liquid and vapor coexist at the same temperature and pressure), and the same will hold for the methanol study here. The temperature of the liquid methanol stream at the time it is probed with the X-ray beam is $\sim 280 \mathrm{~K}$, as determined via vibrational Raman thermometry. ${ }^{19}$

\section{O K Edge: Experiment}

Shown in Figure 2 is the high resolution $(0.1 \mathrm{eV})$ TEY O K edge spectrum of methanol vapor measured $2 \mathrm{~cm}$ from the liquid microjet. Our spectra of molecular methanol agree favorably with those measured by other groups. ${ }^{20,21}$ The peak labeled 1 is generally considered to originate from the 3s Rydberg state with some $\mathrm{O}-\mathrm{H} \sigma^{*}$ character. From the DFT calculations in 


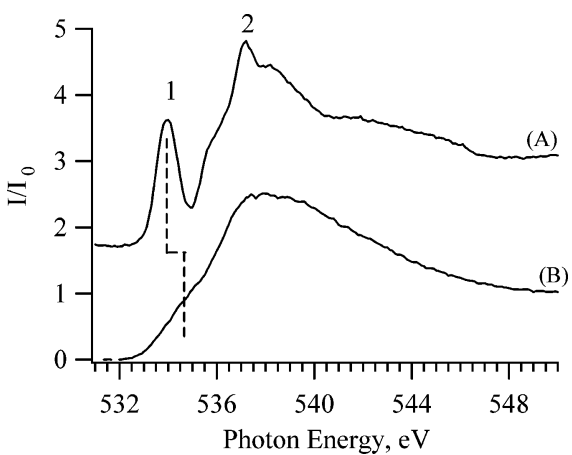

Figure 2. O K edge XA spectra of methanol: TEY (A) vapor and (B) bulk liquid. The two spectra illustrate the spectral changes upon condensation. The peak labeled 1 in the gas phase has been assigned to the $4 \mathrm{a}_{1} \mathrm{O}-\mathrm{H} \sigma^{*}$ antibonding molecular orbital whereas peak 2 is currently assigned to the $\mathrm{C}-\mathrm{O} \sigma^{*}$ state. The vertical line at $534 \mathrm{eV}$ illustrates the region most sensitive to intermolecular hydrogen bonds formed upon condensation.

ref 8 and the present work, however, we assign peak 1 to an orbital of $\sigma^{*}$ character, localized along the internal $\mathrm{O}-\mathrm{H}$ bond. Furthermore, evidence for the antibonding MO character of this orbital arises primarily from studies of the corresponding $4 \mathrm{a}_{1}$ state in water, which has been extensively characterized at the surface of ice by photoion-photoelectron coincidence ${ }^{22}$ techniques, with further support for this assignment provided by the ultrafast bond dissociation phenomena observed by Menzel and co-workers. ${ }^{18,23}$ Nevertheless, as for $\mathrm{H}_{2} \mathrm{O}$, spectral intensity arises primarily from $\mathrm{O} \mathrm{p}_{z}$ orbital contributions to this resonance. The peak at $537 \mathrm{eV}$ (peak 2) has been assigned to 3p Rydberg states, ${ }^{20}$ although our DFT calculations, as described below, show that it should rather be assigned to the $\mathrm{C}-\mathrm{O} \sigma^{*}$ state.

Shown in Figure 2 is the TEY O K edge spectra measured when the photon beam and liquid target are spatially coincident. The most notable effect upon condensation is the significant reduction of the preedge or "threshold" feature (peak 1) at 534 $\mathrm{eV}$ in the gas-phase methanol spectrum. In the deconvoluted TEY spectrum peak 1 is shifted up in energy by $\sim 1 \mathrm{eV}$ and broadened relative to the gas phase appearing merely as a shoulder on the broad band centered at $537 \mathrm{eV}$. The broadening upon condensation of the main spectral feature centered at 537 $\mathrm{eV}$ implies greater orbital overlap with the neighboring molecules within the liquid.

The changes in the intensity of the O K edge "threshold" feature (peak 1) upon condensation can be understood by comparison with the behavior of the corresponding $\mathrm{a}_{1}$ state in the $\mathrm{K}$ edge vapor spectra of isoelectronic $\mathrm{C}, \mathrm{N}$, and $\mathrm{O}$ hydrides. ${ }^{6}$ The $3 \mathrm{a}_{1}$ LUMO orbital in $\mathrm{CH}_{4}$ contains only $\mathrm{s}$ character, resulting from tetrahedral symmetry and therefore exhibits negligible preedge spectral intensity. The small remaining intensity arises from vibronic coupling to the asymmetric stretching vibration of the $\mathrm{CH}_{4}$ molecule, which breaks this symmetry. On the other hand, the p orbitals in $\mathrm{CH}_{4}$, located at higher energy, form a degenerate state of $\mathrm{T}_{\mathrm{u}}$ symmetry. As this tetrahedral symmetry is broken around the central atom, as in molecular $\mathrm{NH}_{3}{ }^{24}$ and $\mathrm{H}_{2} \mathrm{O}^{23}$ shown in Figure 3, larger $\mathrm{p}$ contributions appear in the $\mathrm{a}_{1}$ state, resulting in monotonically increasing spectral intensity that culminates in a giant line resonance for the linear HF molecule, containing almost entirely $\mathrm{F}$ p character. Cavalleri et al. ${ }^{8}$ have used this analogy to describe the changes in the XA spectrum of water upon condensation. Specifically, the absence of significant intensity in the $4 a_{1}$ region of hexagonal ice has been correlated with the near-tetrahedral arrangement (similar to that of $\mathrm{CH}_{4}$ ) of hydrogens and hydrogen bonds to each oxygen atom. Conversely, the liquid water XA
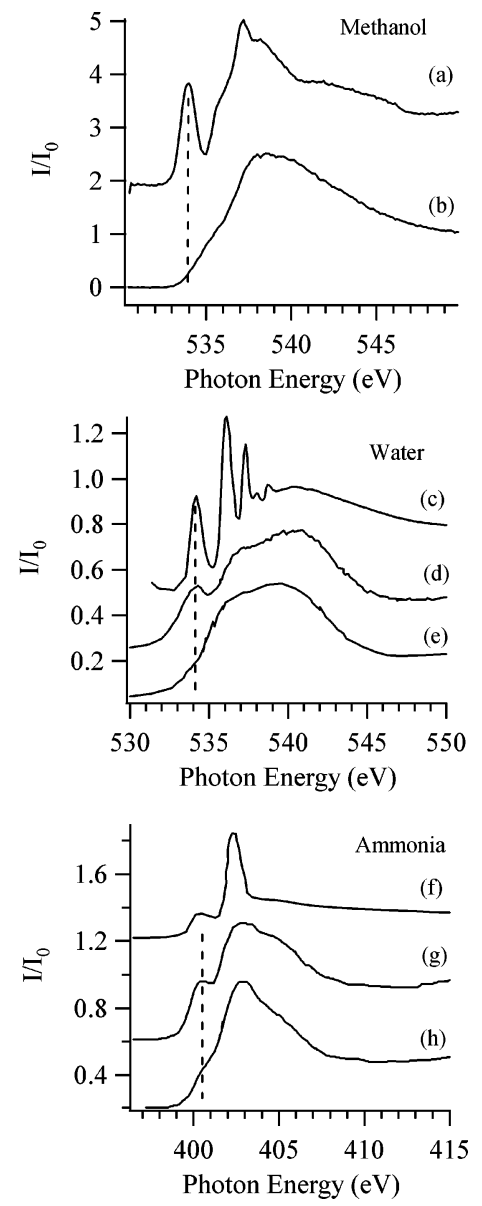

Figure 3. XA spectra of (top) methanol, (middle) $\mathrm{H}_{2} \mathrm{O}$ and (bottom) $\mathrm{NH}_{3}$. The methanol spectra (top) are taken from Figure 2 and correspond to (a) vapor and (b) bulk liquid. The $\mathrm{H}_{2} \mathrm{O}$ spectra are arranged from (d) vapor $^{46}$ (e) surface (f) and bulk amorphous ice measured by Coulman et al. ${ }^{23}$ The $\mathrm{N} \mathrm{K}$ edge ammonia vapor spectrum (g) was measured by Wight and Brion ${ }^{43}$ via electron energy loss whereas the amorphous solid $\mathrm{NH}_{3}$ surface (h) and bulk (i) spectra are reproduced from Rosenberg et al. in ref 24 . In each spectrum the dashed line highlights the $4 a_{1}$ state that exhibits the largest difference from the bulk spectra. The bulk spectra $\left(\mathrm{CH}_{3} \mathrm{OH}, \mathrm{H}_{2} \mathrm{O}, \mathrm{NH}_{3}\right)$ measured via electron yield show similar spectral profiles, which are essentially broadened and blueshifted replicas of the corresponding vapor spectrum.

spectrum exhibits a threshold feature, indicating a disruption in this tetrahedral symmetry by a significant number of broken hydrogen bonds on the donor side of the molecule; termed D-ASYM. ${ }^{8,11,14}$ or single-donor (SD). ${ }^{15}$ This D-ASYM molecular configuration is likened to the isolated methanol molecule, which is an extreme example of this kind of asymmetry.

The absence of intensity in the preedge region of the TEY (bulk) spectrum of liquid methanol thus appears to indicate that the hydrogen bonds within the liquid are arranged in such a way as to increase the overall symmetry around the oxygen atom, effectively removing much of the $\mathrm{O}$ p contribution to the $4 \mathrm{a}_{1}$ resonance. Again, by analogy to water ice, these $\mathrm{p}$ contributions appear in the bulk liquid spectrum ${ }^{8,14,15}$ at higher energies $(\sim 538 \mathrm{eV})$, contributing to the increased intensity just below the IP of liquid methanol. As is also the case for water, the intensity of the $4 a_{1}$ state is found to decrease upon the formation of a hydrogen bond on the donor side of the molecule, as will be discussed below using methanol clusters computed via DFT.

For further comparison, the bulk electron yield XA spectra of amorphous solid ammonia ${ }^{24}$ and water ice, ${ }^{23}$ are shown in Figure 3 . These spectra both exhibit the same overall changes 
in intensity as are observed for the liquid methanol (TEY) when comparing the spectrum of the isolated molecule in the vapor to one embedded in a hydrogen bonding matrix. The $4 \mathrm{a}_{1}$ state (shown in Figure 3 as a vertical line) is significantly perturbed in each case, despite the differences in the number of hydrogen bonding sites and strength $\left(\mathrm{NH}_{3} \mathrm{vs} \mathrm{H}_{2} \mathrm{O}\right)$. All three bulk spectra $\left(\mathrm{CH}_{3} \mathrm{OH}, \mathrm{NH}_{3}\right.$ and $\left.\mathrm{H}_{2} \mathrm{O}\right)$ appear to be broadened and blueshifted replicas of the corresponding resonances in the gas phase.

In each of the spectra $\left(\mathrm{CH}_{3} \mathrm{OH}, \mathrm{H}_{2} \mathrm{O}, \mathrm{NH}_{3}\right)$ the main difference between the surface and bulk is the persistence of some preedge intensity corresponding to the $4 \mathrm{a}_{1}$ state in the vapor. From the above considerations of symmetry, it can be inferred that molecules at the surface of these disordered materials occupy less symmetric hydrogen bonding configurations relative to those molecules residing within the bulk material. Menzel and co-workers ${ }^{18,23,25}$ have conducted an exhaustive study of condensed ammonia, water and methane. They selected these isoelectronic hydrides to isolate the influence of intermolecular hydrogen bonding on electronic structure. $\mathrm{H}_{2} \mathrm{O}$ possesses stronger hydrogen bonds than $\mathrm{NH}_{3}$, whereas dispersion forces dominate intermolecular attraction in solid $\mathrm{CH}_{4}$. For amorphous ice and solid ammonia, they ${ }^{18,23,25}$ concluded that the asymmetry in hydrogen bonding inherent at these amorphous solid interfaces is due to one free $\mathrm{N}-\mathrm{H}$ or $\mathrm{O}-\mathrm{H}$ bond extending away from the surface into vacuum, whereas the other(s) are coordinated to neighboring molecules in the bulk. The net effect is that these bonds become inequivalent, with the preedge $4 \mathrm{a}_{1}$ feature assigned to a new orbital localized along the uncoordinated $\mathrm{O}-\mathrm{H}$ or $\mathrm{N}-\mathrm{H}$ surface bond. ${ }^{25}$ The formation of a localized $\mathrm{O}-\mathrm{H}$ orbital is also observed in the DFT computed spectrum of the D-ASYM species, used in the analysis of the electronic structure of liquid water reported by Cavalleri et al. ${ }^{8}$ Both the D-ASYM species in liquid water and the preedge peak assigned to an uncoordinated $\mathrm{X}-\mathrm{H}$ surface bond in these amorphous ices serve to illustrate the unique XA spectral signatures that allow broken donor hydrogen bonds to be readily identified. ${ }^{14,15}$

In contrast, Scheurer et al. ${ }^{25}$ find that the solid methane surface spectrum exhibits only minimal enhancement of the $a_{1}$ state. The small increase in surface $\mathrm{a}_{1}$ spectral intensity at the solid methane surface was attributed to molecules oriented with one $\mathrm{C}-\mathrm{H}$ bond normal to the surface, in this way polarizing the centrosymmetric $\mathrm{a}_{1}$ orbital perpendicular to the surface, and thus enhancing its p character. No such "threshold" enhancement of the $\mathrm{a}_{1}$ state in solid $\mathrm{NH}_{3}, \mathrm{H}_{2} \mathrm{O}$ or $\mathrm{CH}_{4}$ was observed in the electron yield spectrum, which is sensitive to the bulk electronic structure. This merely indicates that, with the exception of defects, bulk molecules are arranged in symmetric tetrahedral hydrogen bonding configurations. ${ }^{18,23,25}$

The absence of this well-resolved feature at $534 \mathrm{eV}$ in the methanol TEY spectrum would be consistent with fewer broken donor bonds and a larger average number of hydrogen bonds per molecule-consistent with either the cyclic clusters ${ }^{26}$ or longer chain models (7-12 monomers) proposed for liquid methanol. ${ }^{27}$ These assertions will be explored in more detail in section VI "one molecule at a time" using density functional theory (DFT) calculations of simple methanol clusters to better understand the spectral contributions of various ring and linear chain morphologies.

\section{C K Edge: Experiment}

Shown in Figure 4 is the experimental TEY C K edge spectrum of methanol vapor. To our knowledge, there are only two other high-resolution XA studies of molecular methanol,

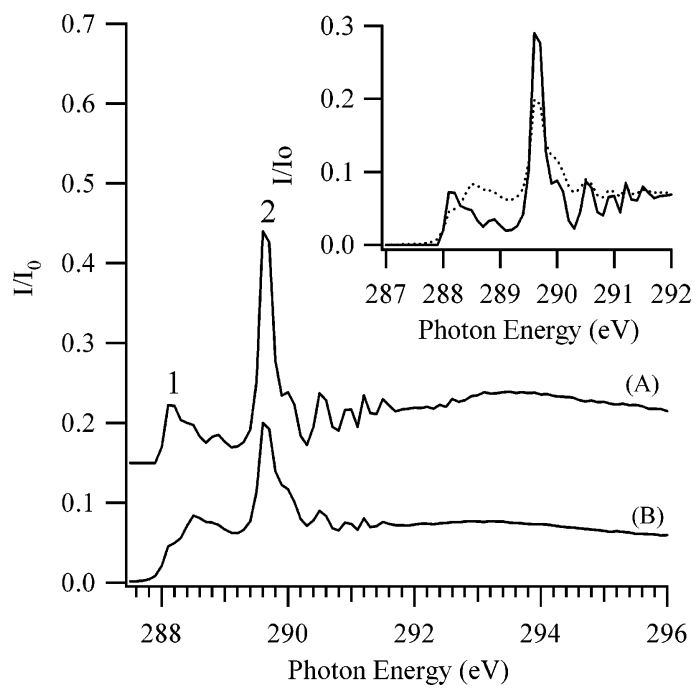

Figure 4. C K edge XA spectra of methanol: TEY (A) vapor, and (B) bulk liquid. Inset: Expanded energy scale showing the fine structure changes at the near edge apparent in the vapor (solid) and bulk (dot) spectra.

those reported by Hempelmann et al. ${ }^{20}$ and Prince et al. ${ }^{28}$ Previous to the present work, there are only the theoretical calculations of the $\mathrm{O} 1 \mathrm{~s}$ inner-shell excitation of methanol reported in ref 8 but no assignment of the excitations was given and, as a result, spectral assignments remain rather controversial. In the present work we have thus recomputed the gas-phase spectrum and will discuss the assignment of the gas-phase features together with the DFT calculations in the next section.

Also shown in Figure 4 is the TEY C K edge spectra of liquid methanol. The TEY spectrum, which is sensitive to the electronic structure of the bulk liquid, exhibits three main differences from the corresponding gas phase spectrum. First, peak 1 in the gas phase, located at $288 \mathrm{eV}$, is markedly decreased in intensity in the TEY (bulk) spectrum, with a concurrent increase in spectral intensity at $288.5 \mathrm{eV}$. Second, peak 2 is reduced in intensity in the bulk TEY spectrum relative to the gas phase. Last, the $\mathrm{C}-\mathrm{O} \sigma^{*}$ resonance $(293 \mathrm{eV})$ is broadened, with the maximum shifted to lower photon energy by $\sim 0.2 \mathrm{eV}$.

The close correspondence of the $\mathrm{C} K$ edge TEY liquid spectra of methanol with that of the vapor could indicate a significant spectral contribution from gas phase molecules immediately surrounding the liquid jet. From the $\mathrm{O} \mathrm{K}$ edge spectra we can estimate, via subtracting the vapor from the TEY surface spectrum, that there is no more than $10 \%$ vapor contribution to the measured ion signal. This is consistent in magnitude with the $10-15 \mathrm{X}$ enhancement of the ion signal on the liquid jet relative to the gas-phase background observed when the microjet is translated out of the photon beam. ${ }^{2}$ The influence of the vapor sheath surrounding the liquid jet is discussed in more detail in ref 19 . We find that the same subtraction procedure applied to the $\mathrm{C} \mathrm{K}$ edge spectra cannot account entirely for the close correspondence between the TEY liquid and vapor spectra. Therefore we are led to the conclusion that the electronic structure around the carbon atom at the liquid methanol surface exhibits minimal perturbation relative to the gas phase. This is consistent with the recent work of Prince et al., ${ }^{28}$ who report that $-\mathrm{CH}$ groups, in a variety of polyatomic molecules, are rather insensitive to intermolecular bonding. For example, the C K-edge spectrum of dimethyl ether ${ }^{28}$ is nearly identical to that of methane and methanol despite the different molecular symmetries. In contrast, the ${ }^{28}$ find that this is not the case for 
spectra of hydroxyl groups, which are strongly influenced by neighboring atoms. Therefore, it may not be surprising that the methyl groups, which are insensitive to the nature of intramolecular bonds, would exhibit relatively little sensitivity to weaker dispersion forces upon condensation. To account for the specific changes in the TEY O and $\mathrm{C} \mathrm{K}$ edge spectra outlined above, DFT calculations on model methanol clusters will now be discussed.

\section{DFT Analysis}

A. Computational Approach. To further interpret the experimental spectral features outlined above, DFT spectrum calculations using simple methanol clusters (linear chains and rings) are used to provide a molecular picture of the HB structure within the liquid and its modification at the surface. The spectral intensity (or oscillator strength) in X-ray absorption is determined by the transition moment between the initial and final states (separated by energy difference $\hbar \omega_{\text {if }}$ ):

$$
I_{\text {if }}=\frac{2}{3} * \omega_{\text {if }}\left|\left\langle\Psi_{\mathrm{i}}|\mu| \Psi_{\mathrm{f}}\right\rangle\right|^{2}
$$

These transition moments can be computed within the transitionpotential approach, which involves the evaluation of a wave function with a half-occupied core-hole. ${ }^{29}$ The same relaxed orbitals are used to describe the initial and final states and the transition element can thus be reduced to a transition involving the core orbital, typically a $\varphi_{1 \mathrm{~s}}$ orbital, and an unoccupied orbital $\varphi_{\mathrm{f}}$ :

$$
I_{\text {if }}=\frac{2}{3} * \omega_{\mathrm{f}}\left|\left\langle\varphi_{1 \mathrm{~s}}|\mu| \varphi_{\mathrm{f}}\right\rangle\right|^{2}
$$

The use of a half-occupied core hole treats relaxation effects up to second order and reproduces the absolute transition energies to within $1-2 \mathrm{eV}$ of the experimental values; ${ }^{30}$ the accuracy can be further improved to better than $0.5 \mathrm{eV}$ by specifically relaxing each core-excited state. ${ }^{31}$ Because of the dipole selection rule, ${ }^{6}$ the transition moment involving excitation from the 1s core-level will be sensitive to the local p-character in the excited orbital.

These calculations give as result a discrete set of energy levels, each of which is associated with an oscillator strength and a line broadening. The experimental broadening of a transition below the edge has contributions from instrumental, lifetime as well as vibrational (Franck-Condon) broadening, which will be mimicked in the theoretical spectra by convoluting with Gaussian functions of the appropriate full width at halfmaximum (fwhm); for the continuum we apply a larger broadening. The computed $\mathrm{O} \mathrm{K}$-shell spectra are thus broadened using Gaussian functions of constant width of $0.8 \mathrm{eV}$ up to 537.5 $\mathrm{eV}$ and then linearly increased to $8 \mathrm{eV}$ until $550 \mathrm{eV}$, as used in ref 14 where it has been shown to reproduce effectively the experimental hexagonal ice XAS spectrum. The C K-edge spectra are convoluted using Gaussian functions with constant fwhm of 0.5 up to $292 \mathrm{eV}$ and then linearly increased to 0.7 $\mathrm{eV}$ in a $20 \mathrm{eV}$ range. Using this approach, we find excellent agreement between the experimental vapor and computed monomer spectra. as shown in Figure 5.

XA spectra are computed using the molecular orbital DFT StoBe deMon code. ${ }^{32}$ In the present work the system is modeled as bare clusters (rings or linear chains), where the interaction with the rest of the liquid is not taken into account. The effects of hydrogen bond formation on the electronic structure of the methanol molecule are quite local in nature and can be
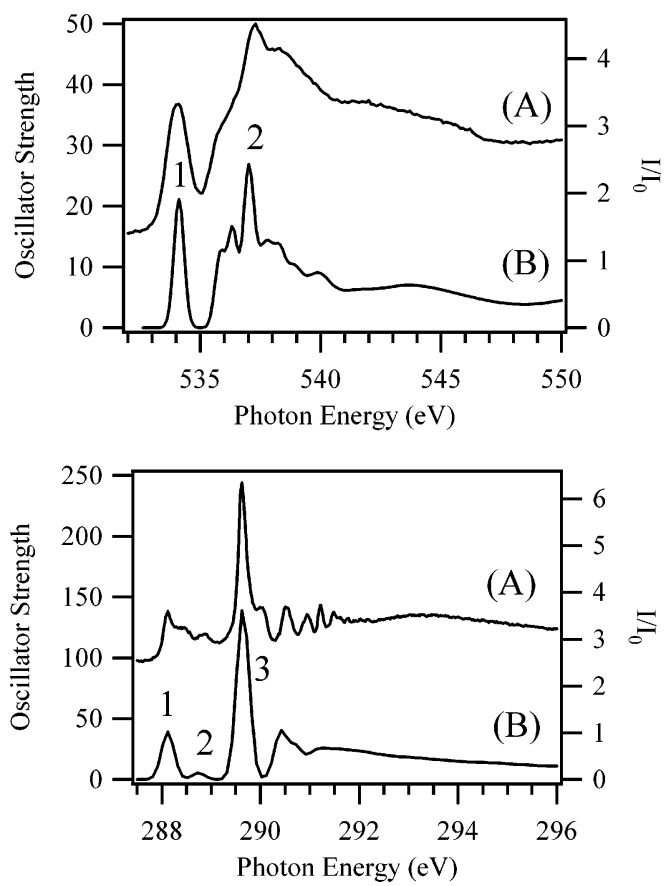

Figure 5. Methanol vapor spectra measured at the $\mathrm{O}$ (top, $\mathrm{A}$ ) and $\mathrm{C}$ $\mathrm{K}$ (bottom, A) edges. DFT computed spectra for the methanol monomer at the $\mathrm{O}$ (top, B, from ref 8) and $\mathrm{C} \mathrm{K}$ (bottom, B) edges.

effectively described within this framework. Different topologies are considered for the methanol clusters: chains up to the 15mer, and rings up to six-members. The $\mathrm{O}-\mathrm{O}$ distance between neighboring molecules is taken to be either $2.62 \AA$, as determined previously for the surface, ${ }^{4}$ or $2.7 \AA$, as determined for the bulk. ${ }^{4}$ We find that the computed spectra, using the surface $\mathrm{O}-\mathrm{O}$ distance, exhibit spectral profiles nearly identical to those computed using a liquid distance of $2.7 \AA$, which greatly simplifies the overall conclusions drawn from these DFT calculations.

The methanol molecule has an intramolecular $\mathrm{O}-\mathrm{H}$ distance of $0.945 \AA$, the $\mathrm{C}$ atom is connected to the $\mathrm{O}$ and to the hydrogens in the methyl group with distances of 1.425 and 1.1 $\AA$, respectively, and the $\mathrm{C}-\mathrm{O}-\mathrm{H}$ angle is $108.5^{\circ}$. For the dimer the angle between the intramolecular $\mathrm{O}-\mathrm{H}$ bond and the hydrogen bond direction is about $157^{\circ}$. This geometry is reasonably close to those obtained by ab initio quantum chemical calculations. $^{33}$ In theoretical cluster model calculations one approach is to perform geometry optimization for each cluster size. In this manner a self-consistent description of all geometrical parameters is obtained at the chosen level of theory. However, the changes in the resulting XA spectra arise both from the change in the geometry and in the hydrogen bond topology. By keeping the geometry fixed as a function of cluster size, we eliminate the effects of internal structure changes, while studying the effect of the different $\mathrm{HB}$ configurations in the liquid. Furthermore, for these types of hydrogen bonding systems, consisting of several molecules, a large number of local minima, with energies not high above the global minimum can be found. For example, this has been shown by Franken et al. ${ }^{34}$ for the six-member water ring, and it can be assumed to be even more true at the elevated temperatures characteristic of molecular liquids. The appropriate choice of geometry is less than straightforward and therefore we have chosen to study an idealized situation.

The core-excited atom is described using the IGLO-III allelectron basis set, ${ }^{35}$ allowing for the relaxation of the core orbitals. Effective core potentials $(\mathrm{ECP})^{36}$ are used on all other 

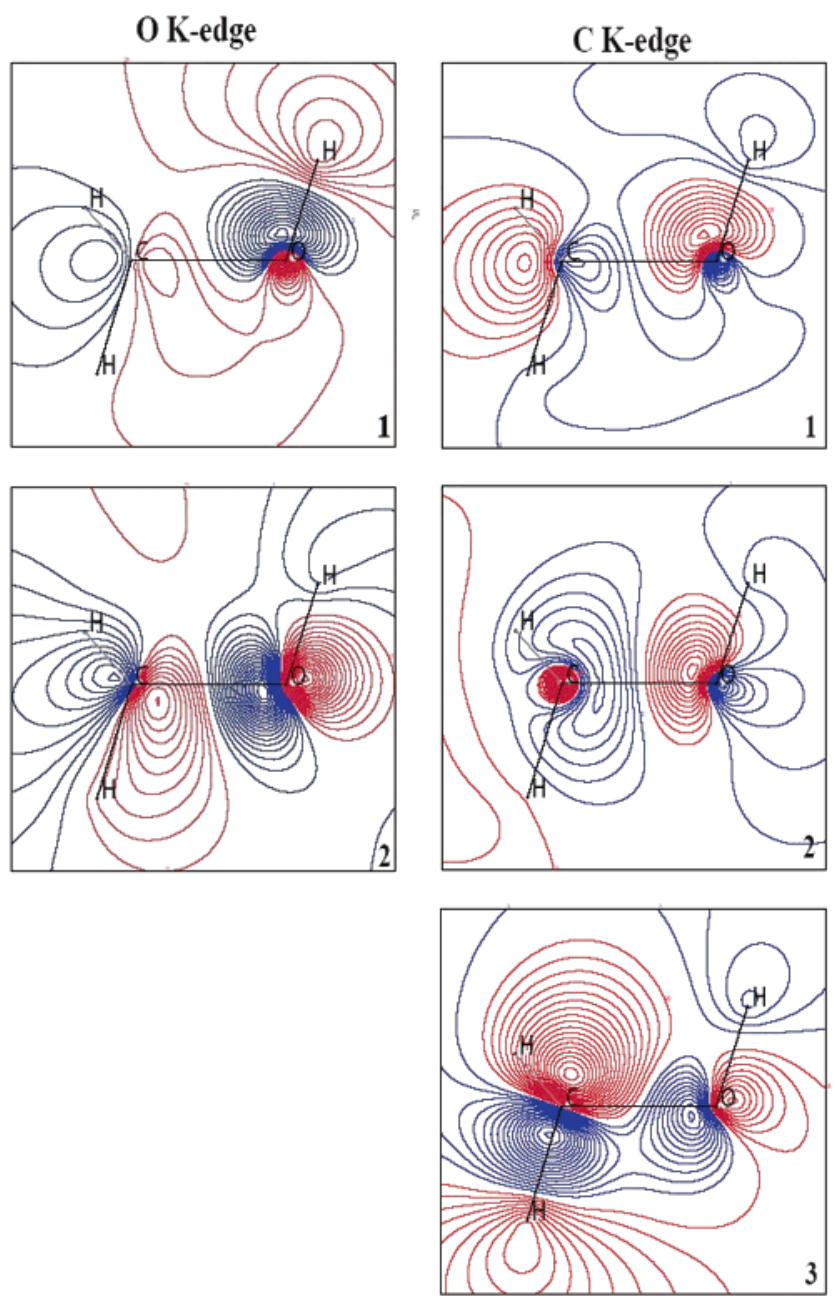

Figure 6. Contour plots of the excited orbitals corresponding to the main spectral features (labeled $1-3$ in Figure 5) of the methanol monomer. The orbitals are plotted in the $\mathrm{C}-\mathrm{O}-\mathrm{H}$ plane.

atoms of the excited type ( $\mathrm{C}$ or $\mathrm{O}$ ) to simplify the definition of the core hole. The calculations are performed using a doublebasis-set technique where, in the spectrum calculation, the normal molecular basis is augmented by a large diffuse basis set $(\approx 150$ functions) to improve the description of the higherlying Rydberg and continuum states. ${ }^{37}$ For all cluster calculations nonlocal exchange and correlation functionals developed by Becke, ${ }^{38}$ Perdew and Wang ${ }^{39}$ are used.

B. DFT: Gas Phase. The geometry of the methanol monomer was optimized at the DFT/B3LYP ${ }^{40,41}$ level using the Gaussian 98 program package. ${ }^{42}$ For the geometry optimization, the standard 6-31G basis with diffuse functions was used on all atoms. The computed $\mathrm{O}$ K-edge spectrum for the methanol monomer is shown and compared with experiment in Figure 5. For this small molecule the theoretical spectrum has been calculated using the transition potential technique with relativistic and differential relaxation effects ${ }^{31}$ explicitly included. The relativistic and relaxation corrections computed this way for the gas-phase molecule are then used to correct the position of all simulated O-edge spectra of the more extended cluster models. In Figure 6 orbital contour plots are shown in the $\mathrm{C}-\mathrm{O}-\mathrm{H}$ plane of the excited molecule. The orbital labeled as 1 in the $\mathrm{O} \mathrm{K}$-edge spectra in Figure 5 can be identified with the $(\mathrm{O}-\mathrm{H}) \sigma^{*}$ orbital, as discussed in the previous section, with some contribution from the $\mathrm{p}_{z}$ orbital centered on the $\mathrm{C}$-atom. The nature of this orbital makes its dependence on condensation evident. The peak labeled 2 has instead clear $(\mathrm{C}-\mathrm{O}) \sigma^{*}$ character.
The C-edge spectrum of the gas phase molecule has been computed fully within the transition potential approach and then shifted to align its third sharp feature under the correspondent experimental peak at $289.8 \mathrm{eV}$ (peak 2 in Figure 4). Because of its nature, this peak, whose position is unaffected by condensation, is a good candidate for calibration of the theoretical spectra so that we aligned in the same way as for the gas-phase all C-edge spectra computed for bigger clusters.

The assignment of the spectral features in the C-edge XA spectrum of methanol is more controversial. The resonance $(288.0 \mathrm{eV})$ labeled peak 1 in Figure 4 has been previously assigned ${ }^{43}$ to the $2 \mathrm{a}^{\prime \prime} \rightarrow 3$ s Rydberg state. Later it was argued, ${ }^{44}$ by analogy with the alkanes, that this transition originates from excitation to a valence $(\mathrm{C}-\mathrm{H}) \sigma^{*}$ orbital. Orbital contour plots of the methanol monomer with core-excited carbon are shown in Figure 6. Peak 1 (Figure 5) can be assigned to the same molecular orbital of the preedge peak in the $\mathrm{O}$ edge spectrum, with a $\mathrm{p}$ orbital of the carbon mixing with the $(\mathrm{O}-\mathrm{H}) \sigma^{*}$. The progression of peaks, in the experimental spectrum (Figure 5), slightly shifted to higher energies from peak 1 , is due to unresolved vibrational structure due to either the $\mathrm{C}-\mathrm{O}$ (120 $\mathrm{meV})$ or $\mathrm{C}-\mathrm{H}(340 \mathrm{meV})$ vibrational modes. The peak with relatively small intensity at $288.8 \mathrm{eV}$ can instead be assigned to the antibonding $(\mathrm{C}-\mathrm{O}) \sigma^{*}$ orbital, as shown in Figure 6 .

A similar controversy exists for the assignment of peak 2 in Figure 4, which was first thought ${ }^{43}$ to be due to a $3 \mathrm{a}^{\prime \prime}$ Rydberg state but later assigned ${ }^{44}$ to a $(\mathrm{C}-\mathrm{H}) \sigma^{*}$ resonance. This latest assignment is confirmed by the DFT calculation of the gasphase molecule, as shown in Figure 6 (orbital 3); the $(\mathrm{C}-\mathrm{H})$ $\sigma^{*}$ orbital is heavily mixed with a carbon p-orbital. The third broad feature at $293.2 \mathrm{eV}$ has been assigned to the $\mathrm{C}-\mathrm{O} \sigma^{*}$ resonance. ${ }^{44}$ In reality, as for molecular $\mathrm{H}_{2} \mathrm{O}$, these resonances undoubtedly arise from excitations into states of mixed Rydberg/ valence character.

C. DFT: Linear Chains. Shown in Figure 7 is a set of computed spectra (O K edge) for each methanol molecule in a seven-member linear chain. Molecules (labeled B-F) reside in the interior of the linear chain and have a coordination number of 2; each molecule accepts and donates a hydrogen bond. The terminal molecules (A and $\mathrm{G}$ ) have a coordination number of 1 due to the broken donor or acceptor hydrogen bond. In this way, we can evaluate the spectral differences between molecules with 1 and 2 hydrogen bonds one molecule at a time. Particular attention is paid to the computed spectra of the terminal molecules (A and $\mathrm{G}$ ), which contain a broken hydrogen bond. Molecules B-F exhibit very similar spectral profiles with the onset of appreciable oscillator strength at $535 \mathrm{eV}$. In contrast, the terminal molecules (A and $G$ ) show a much lower threshold with a peak centered at $534 \mathrm{eV}$. The dashed line in Figure 7 illustrates this $+1 \mathrm{eV}$ shift of preedge oscillator strength, clearly indicating the sensitivity of XA to both broken donor and acceptor hydrogen bonds in this system. Although, we cannot readily distinguish experimentally between different interior molecules, the terminal methanol molecules provide a clear "fingerprint" in the low energy threshold feature at $534 \mathrm{eV}$. The $+1 \mathrm{eV}$ shift of the first preedge peak in the computed spectra of the interior and terminal molecules quantitatively reproduces the shift and energy position observed in the $4 \mathrm{a}_{1}$ peak in both the gas and bulk experimental spectra.

Also shown in Figure 7 is the analogous treatment of the $\mathrm{C}$ $\mathrm{K}$ edge spectra using the same seven-member chain. The computed spectra of the interior molecules $(B-F)$ exhibit spectral profiles more complicated than those of the $\mathrm{O} \mathrm{K}$ edge, yet the terminal molecule (A) with the broken donor bond 

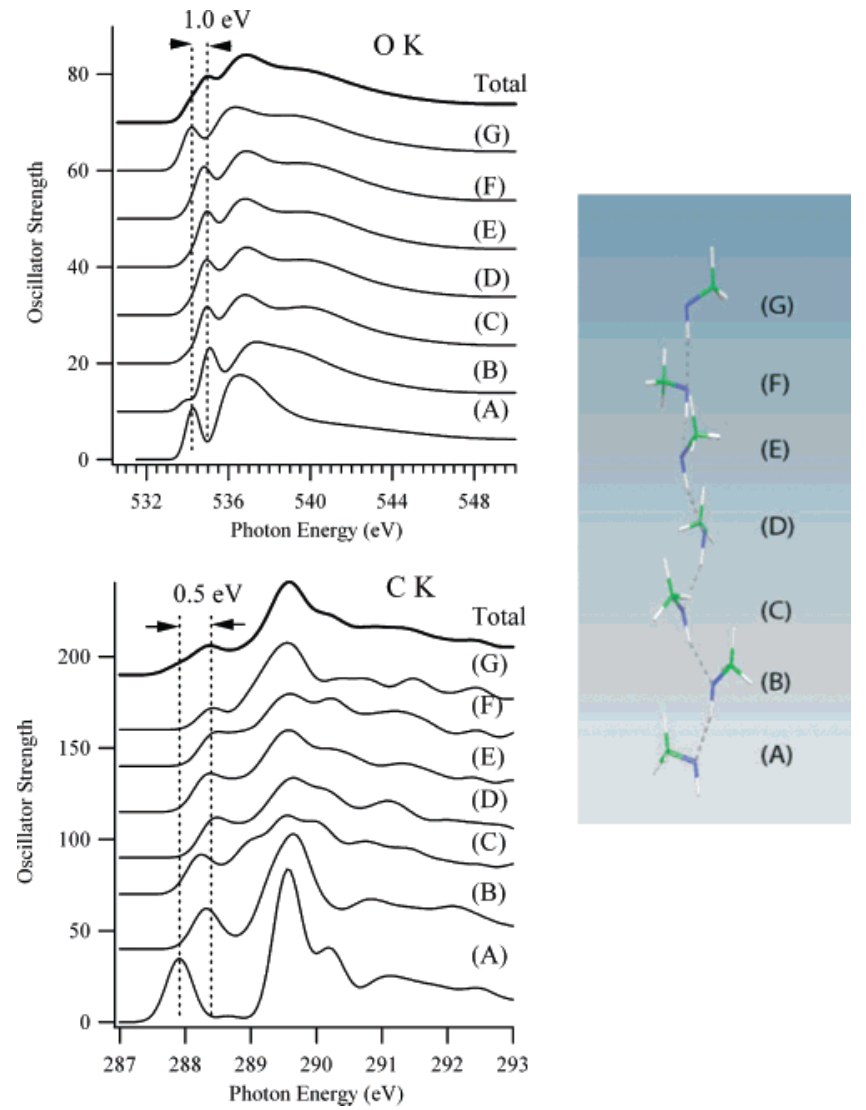

Figure 7. DFT computed $\mathrm{O}$ (top) and $\mathrm{C}$ (bottom) K edge spectra for molecules in a 7-member linear chain. Spectra labeled $(\mathrm{B}-\mathrm{F})$ are computed for molecules in the interior portion of the chain each with 2 H-bonds. Spectra A and G are computed for terminal molecules residing at the end of the chain with either a broken donor or acceptor hydrogen bond. The total spectrum shown is merely the average of single molecule spectra $A-\mathrm{G}$.

exhibits a preedge peak shifted by $0.5 \mathrm{eV}$ to lower energies (shown with a dashed line). This low energy feature is absent in computed spectra of the interior molecules $(\mathrm{B}-\mathrm{F})$ as well as the opposing terminal donor-only molecule labeled G. The computed shift $+0.5 \mathrm{eV}$ of the interior molecule relative to the computed methanol monomer (shown in Figure 5) spectrum is consistent with the gas-to-bulk shift of $+0.5 \mathrm{eV}$ determined experimentally. This implies that the bulk liquid is primarily composed of molecules that both donate and accept a hydrogen bond.

To further explore the effects of chain length, spectra were generated by summing up the oscillator strengths for each molecule in various chain lengths to produce total or average spectra, like that shown in Figure 7 for a 7-molecule linear chain. For simplicity, we have built up spectra of various chain lengths by constructing average spectra using the central molecule (D in Figure 7) and the two terminal molecules ( $\mathrm{G}$ and $\mathrm{A}$ in Figure 7). Thus, the spectra of a 9-molecule chain is computed using the approach:

$$
9 \text {-mer }=(7 * \mathrm{D}+1 * \mathrm{~A}+1 * \mathrm{G}) / 9
$$

This building block approach greatly alleviates computational expense and yields results nearly identical to those obtained by explicitly calculating the spectra of each molecule in a given chain. The validity of this building-block approach to generate spectra is demonstrated for a 9-molecule chain in the insets in Figures 8 and 9, where we compare the spectra computed for each atom in the chain with the prediction using spectra from

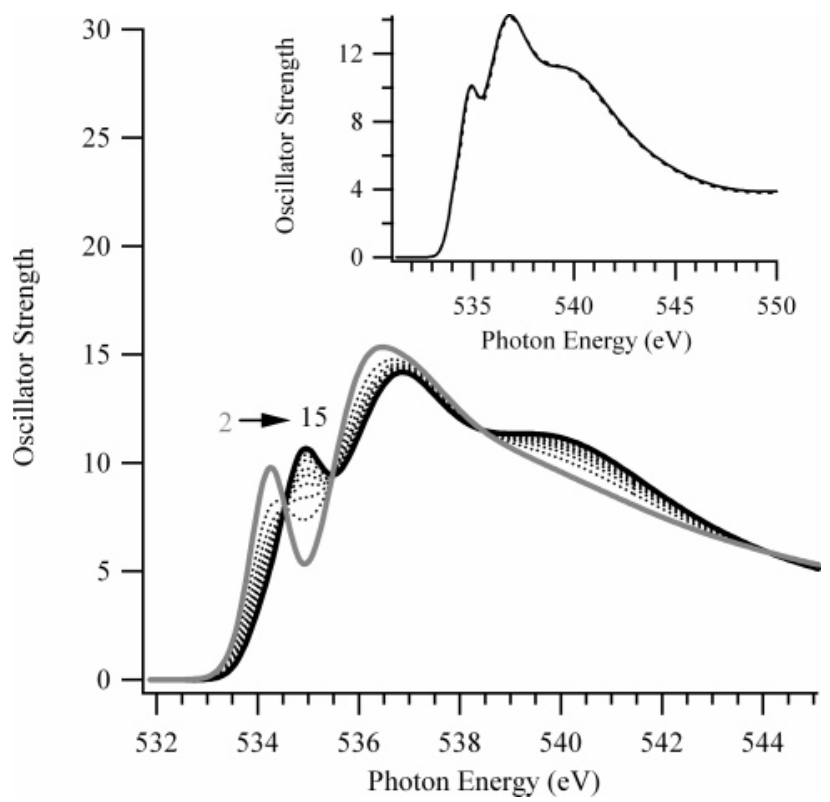

Figure 8. $\mathrm{O} \mathrm{K}$ edge composite spectra showing the evolution of oscillator strength with chain length $\left(\left(\mathrm{CH}_{3} \mathrm{OH}\right)_{n}\right)$. Sizes range from the dimer (gray) to the 15-mer (black). Inset: Computed composite spectra of a 9-mer linear chain using the building block approach (solid) vs explicitly calculating (dot) the spectra of each molecule in the 9-member chain.

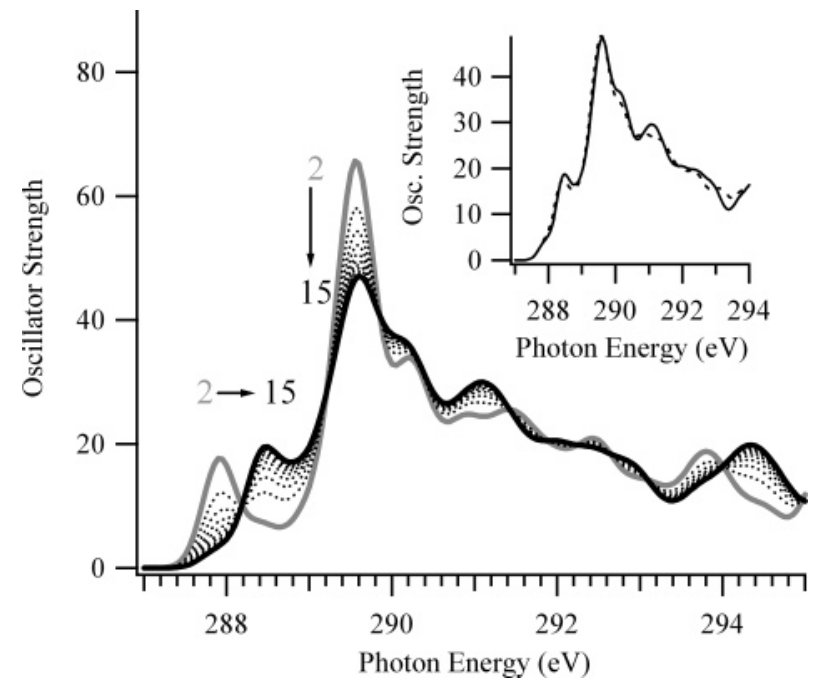

Figure 9. $\mathrm{C} \mathrm{K}$ edge composite spectra showing the evolution of oscillator strength with chain length $\left(\left(\mathrm{CH}_{3} \mathrm{OH}\right)_{n}\right)$. Sizes range from the dimer (gray) to the 15-mer (black). Inset: Computed composite spectra of a 9-mer linear chain using the building block approach (solid) vs explicitly calculating (dot) the spectra of each molecule in the ninemember chain.

interior and terminating molecules as building-blocks. Using this approach, we can easily generate spectra for varying chain lengths and quantify spectral changes as function of increasing chain length.

$\mathrm{O} \mathrm{K}$ edge spectra of chains ranging in size from 2 (dimer) to 15 molecules long are shown in Figure 8. At the O K edge, the most dramatic change in computed oscillator strength with chain length is in the position of the preedge feature corresponding to the $4 \mathrm{a}_{1}$ state in methanol vapor. This preedge feature shifts up in energy as the methanol chain lengthens. Only subtle changes are observed from extending the chain length beyond 7 molecules, with an overall shift of $1.0 \mathrm{eV}$ for long chains relative to the dimer and monomer. In addition, the broad feature centered at $540 \mathrm{eV}$ increases in oscillator strength, again 


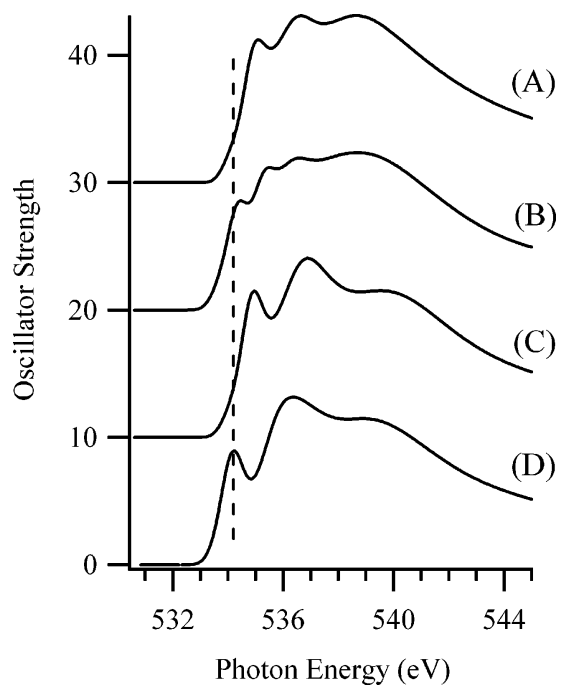

Figure 10. DFT computed $O \mathrm{~K}$ edge spectra of (A) 3- and (B) 6-member cyclic methanol rings. For comparison, the interior molecule (C) and terminal donor (D) (labeled D and G in Figure 7, respectively) from the 7-molecule chain are shown. The dashed line indicates the preedge peak position in the terminal molecule at $534 \mathrm{eV}$.

exhibiting saturation for chains longer than 7 molecules. Upon further inspection the shift of the preedge $4 a_{1}$ feature with chain length merely reflects the shrinking statistical influence of the terminal molecules (with broken $\mathrm{H}$ bonds) for chains longer than 6 molecules. This is illustrated for the case of the trimer in which two-thirds of the molecules occupy terminal positions and therefore dominate the total oscillator strength at $534 \mathrm{eV}$.

The same type of analysis for the C K edge is shown in Figure 9. It can be concluded that with increasing chain length the main spectral changes are 2-fold. First, there is an overall shift in oscillator strength of the $\mathrm{O}-\mathrm{H} \sigma^{*}+\mathrm{C}(\mathrm{p})$ resonance $(288 \mathrm{eV})$ to higher energy. For a 7-mer this shift is $+0.5 \mathrm{eV}$ relative to the dimer. Second, there is a gradual reduction in oscillator strength with increasing chain length of the peak corresponding to the $\mathrm{C}-\mathrm{H} \sigma^{*}$ state in the methanol monomer. As for the $\mathrm{O} \mathrm{K}$ edge spectra, the computed oscillator strengths are nearly identical for chains 7-15 molecules in length.

From the preceding DFT analysis, the experimental O K edge TEY spectrum exhibits a $1 \mathrm{eV}$ shift to $535 \mathrm{eV}$ of the $4 \mathrm{a}_{1}$ resonance, which can be accounted for by the computed spectra of longer chains $(n>6)$ in which molecules with two hydrogen bonds, located in the interior of a chain, dominate the total computed oscillator strength.

D. DFT: Rings. Spectra were also computed for 3- and 6-member rings, in an effort to evaluate their contribution to the experimental spectra. Shown in Figure 10 are the results for the $\mathrm{O} \mathrm{K}$ edge. The computed spectrum for the 3-molecule ring (Figure 10a) exhibits an onset of oscillator strength at 535 $\mathrm{eV}$. The computed spectrum of the 6-member ring (Figure 10b) exhibits a similar overall spectral profile as the 3-molecule ring with a slight shift to lower energy $(534.5 \mathrm{eV})$ of the first preedge peak. This observation is consistent with the resonantly excited $\mathrm{X}$-ray emission spectrum of liquid methanol measured recently by Guo et al., ${ }^{45}$ who find that the onset of absorption of 6-member rings occurs at lower photon energies than do 8 molecule linear chains. For comparison the computed spectrum of the center molecule ( $\mathrm{E}$ in Figure 7 ) as well as a terminal molecule ( $\mathrm{G}$ in Figure 7) in a 7-member linear chain are shown in Figure 10c,d, respectively. Clearly, the terminal molecule (Figure 10d) exhibits a well-defined preedge feature at $534 \mathrm{eV}$ that is absent in both the 3-and 6-molecule rings as well as the

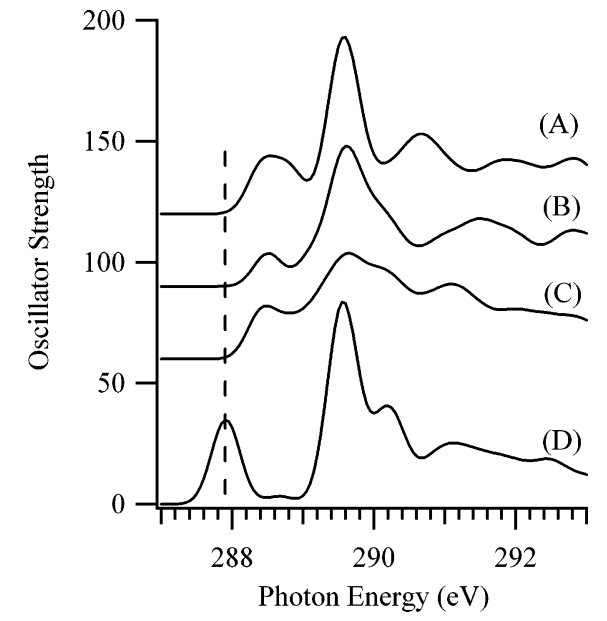

Figure 11. DFT computed C K edge spectra of (A) 3- and (B) 6-member cyclic methanol rings. For comparison, the interior molecule (C) and terminal acceptor (D) (labeled D and A in Figure 7, respectively) of the 7-molecule chain are shown. The dashed line indicates the preedge peak position in the terminal molecule at 288 $\mathrm{eV}$.

interior chain molecule. Despite the obvious differences at higher energies $(537 \mathrm{eV})$, this comparison clearly shows that methanol molecules with 2 hydrogen bonds, whether in a ring or chain interior, lack the well-defined preedge feature at $534 \mathrm{eV}$ clearly evident in the terminal molecule spectrum. Although this comparison suggests that it may indeed be difficult to experimentally distinguish between rings and long chains by XAS, a clear signature remains of terminal molecules and consequently short linear chains.

Shown in Figure 11 is the same analysis as discussed above applied to the $\mathrm{C}$ K edge. Parts a and b of Figure 11 are spectra computed for 3- and 6-member rings, respectively. Included for comparison is an interior molecule ( $2 \mathrm{H}$-bonds) as well as the terminal molecule (1 H-bond, "acceptor only") from a linear 7 -molecule chain. Spectra $11 \mathrm{a}$ and $11 \mathrm{~b}$ exhibit a shift of 0.5 $\mathrm{eV}$ of the first preedge relative to the analogous feature in the terminal molecule spectrum (Figure 11d), whereas spectrum 11c shows a strong reduction in the intensity in this region. Again, these calculations suggest that spectra computed for methanol molecules engaging in $2 \mathrm{H}$-bonds, whether in a ring or in the interior of a chain, all exhibit a $+0.5 \mathrm{eV}$ shift in the $\mathrm{O}-\mathrm{H} \sigma^{*}$ $+\mathrm{C}(\mathrm{p})$ peak position relative to the terminal acceptor molecule.

\section{Conclusions}

From the preceding analysis it is clear that the DFT computed spectra reveal the sensitivity of XAS to broken hydrogen bonds, as already observed in studies of $\mathrm{H}_{2} \mathrm{O} \cdot{ }^{3,11,14}$ Specifically, the $\mathrm{O}$ $\mathrm{K}$ edge computed spectra of terminal molecules show a welldeveloped preedge feature that coincides in energy with the $4 a_{1}$ monomer resonance. Conversely, molecules in the interior of a chain or ring have 2 hydrogen bonds and exhibit the same preedge feature but shifted up in energy by $\sim 1 \mathrm{eV}$ relative to the terminal molecule spectra. This difference leads to a sequential shift of this feature with chain length that merely reflects the greater contribution of 2 hydrogen-bonded molecules in the interior of longer linear chains to the total $n$-mer spectrum. Therefore, we can account for the experimental changes in the $\mathrm{O} \mathrm{K}$ edge spectra by comparing the vapor with the computed monomer, and the bulk (TEY) with longer linear chains $(n>$ 6) or 3, 6-member rings. This comparison is shown in Figure 12. The $\mathrm{O} \mathrm{K}$ edge computed spectra account for the qualitative behavior of the preedge, which is observed to first decrease in 

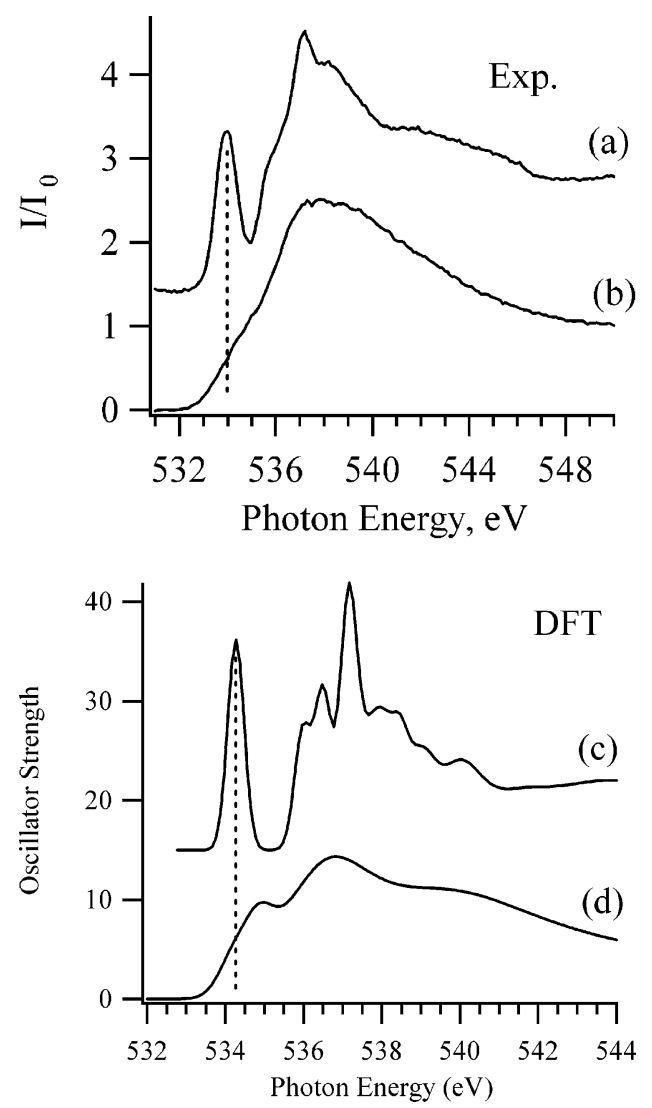

Figure 12. A comparison of the $\mathrm{O} K$ edge experimental (A) vapor and (B) bulk spectra with the DFT computed methanol (C) monomer and (D) linear 7-mer. The dashed lines indicate the position of the $4 a_{1}$ feature in the vapor (or monomer) spectra. The spectral changes in this peak observed in the experiment are well modeled by DFT through an increase in chain length from the monomer (vapor) to a chain of 7 molecules in length (bulk).

oscillator strength in the formation of a linear trimer relative to the monomer and shift by $+1 \mathrm{eV}$ in the computed 7-mer linear chain or ring. In this way, these DFT results can be used to rationalize the spectral changes observed experimentally upon condensation.

It is interesting to compare the development of the $\mathrm{O} \mathrm{K}$ edge spectrum of methanol upon $\mathrm{H}$-bond formation with that of water. In water there are two equivalent $\mathrm{H}$-donating possibilities, and with both coordinated, the preedge feature is quenched, as discussed previously. ${ }^{8}$ The preedge arises when the neartetrahedral symmetry of water in ice is broken and one donating $\mathrm{H}$-bond is lost; the extreme case corresponds to one strong (short) donating H-bond and one broken, similar to gas-phase methanol. However, in methanol the asymmetry is inherent through the strong covalent $\mathrm{C}-\mathrm{O}$ bond and the more weakly $\mathrm{H}$-bonded $\mathrm{O}-\mathrm{H}$. Thus, for methanol the preedge is quenched to a lesser extent through $\mathrm{H}$-bond formation because this bond cannot compete in strength with the internal bonds in the molecule. We find an upward shift in energy of the preedge peak for the interior molecules in the chains, but a rather small loss of intensity due to the inherent asymmetry around the oxygen in this case.

Figure 13 shows the experimental $\mathrm{C} \mathrm{K}$ edge spectra of the vapor and bulk, compared with the computed monomer, and linear 7-mer. Again, an overall gas-to-bulk shift of the first preedge feature by $+0.5 \mathrm{eV}$ is quantitatively captured by the computed monomer to 7-mer spectra. It should also be pointed out that the same shift is observed for the computed 3, 6 ring spectra and therefore we cannot distinguish between the two
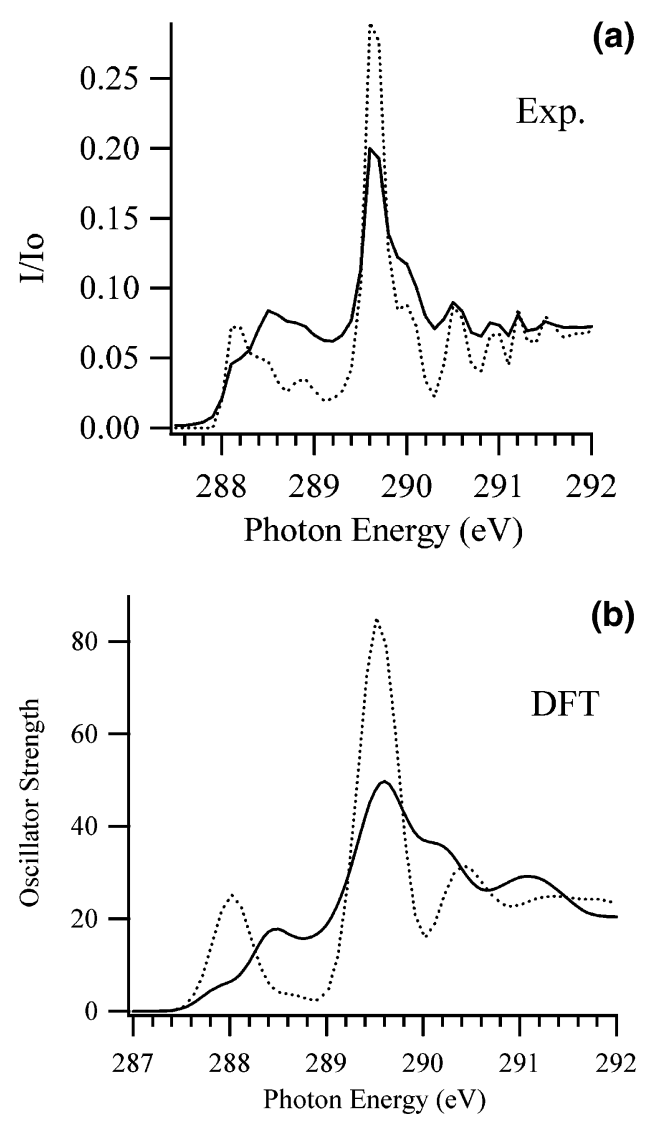

Figure 13. Comparison of the $\mathrm{C} K$ edge experimental (top) vapor (dot) and bulk (solid) spectra with the DFT computed (bottom) methanol monomer (dot), and linear 7-mer (solid). The spectral changes observed in the experiment are well modeled by DFT through an increase in chain length from the monomer (vapor) to trimer a chain of 7 molecules in length (bulk).

possibilities experimentally. The computed linear trimer exhibits spectral changes "intermediate" between the monomer and 7-mer. Last, the two computed spectra shown (monomer and 7-mer) account for the reduction in intensity of the $\mathrm{C}-\mathrm{H} \sigma^{*}$ state observed in the experimental vapor and bulk spectra.

In conclusion, the computed spectra of the 7-15-member linear chain and 6-molecule ring accurately reproduce the spectral changes observed in the TEY and are consistent with the recent XES study, which found evidence for equimolar mixtures of 8 -molecule linear chains and six-member rings. ${ }^{45}$ The DFT computed spectra clearly illustrate the sensitivity in this system to extended methanol morphology (chains and rings). In addition, the terminal molecules exhibit clear differences from those molecules within a ring or chain interior. Surface tension measurements as well as MD simulations both indicate that methanol molecules located at the liquid-vapor interface will be oriented such that the methyl groups extend away from the bulk liquid. These configurations will naturally imply an interfacial layer of terminal methanol molecules. Therefore, on the basis of the DFT calculations presented here, we predict that any changes in methanol chain morphology at the liquidvapor interface should be readily observed in the intensity and position of the preedge $4 a_{1}$ resonance.

Acknowledgment. The experimental work was supported by the Chemical Sciences Division of the US Department of Energy. The theoretical work was supported by the Swedish Foundation for Strategic Research and the Swedish Natural Science Research Council. Generous grants of computer time 
at the Swedish National Supercomputer Center and Center for Parallel Computing are gratefully acknowledged.

\section{References and Notes}

(1) Dang, L. X.; Chang, T. J. Chem. Phys. 2003, 119, 9851.

(2) Wilson, K. R.; Rude, B. S.; Catalano, T.; Schaller, R. D.; Tobin, J.

G.; Co, D. T.; Saykally, R. J. J. Phys. Chem. B 2001, 105, 3346.

(3) Wilson, K. R.; Cavalleri, M.; Rude, B. S.; Schaller, R. D.; Nilsson, A.; Pettersson, L. G. M.; Goldman, N.; Catalano, T.; Bozek, J. D.; Saykally, R. J. J. Physics-Condens. Matter 2002, 14, L221.

(4) Wilson, K. R.; Schaller, R. D.; Co, D. T.; Saykally, R. J.; Rude, B. S.; Catalano, T.; Bozek, J. D. J. Chem. Phys. 2002, 117, 7738.

(5) Kuo, I.; Mundy, C. J. Science 2004, 303, 658.

(6) Stöhr, J. NEXAFS Spectroscopy; Springer-Verlag: Berlin; New York, 1992.

(7) Nilsson, A. J. Electron Spectrosc. Relat. Phenom. 2002, 126, 3.

(8) Cavalleri, M.; Ogasawara, H.; Pettersson, L. G. M.; Nilsson, A.

Chem. Phys. Lett. 2002, 364, 363.

(9) Bluhm, H.; Ogletree, D. F.; Fadley, C. S.; Hussain, Z.; Salmeron, N. J. Phys.-Condens. Matter 2002, 14, L227.

(10) Parent, P.; Laffon, C.; Mangeney, C.; Bournel, F.; Tronc, M. J. Chem. Phys. 2002, 117, 10842.

(11) Bergmann, U.; Wernet, P.; Glatzel, P.; Cavalleri, M.; Pettersson,

L. G. M.; Nilsson, A.; Cramer, S. P. Phys. Rev. B 2002, 6609, U30.

(12) Björneholm, O.; Federmann, F.; Kakar, S.; Möller, T. J. Chem. Phys. 1999, 111, 546.

(13) Bowron, D. T.; Krisch, M. H.; Barnes, A. C.; Finney, J. L.; Kaprolat, A.; Lorenzen, M. Phys. Rev. B 2000, 62, R9223.

(14) Myneni, S.; Luo, Y.; Näslund, L. A.; Cavalleri, M.; Ojamäe, L.; Ogasawara, H.; Pelmenschikov, A.; Wernet, P.; Väterlein, P.; Heske, C. Hussain, Z.; Pettersson, L. G. M.; Nilsson, A. J. Phys.-Condens. Matter 2002, 14, L213.

(15) Wernet, P.; Nordlund, D ; Bergmann, U.; Cavalleri, M.; Odelius, M.; Ogasawara, H.; Näslund, L. A.; Hirsch, T. K.; Ojamäe, L.; Glatzel, P.; Pettersson, L. G. M.; Nilsson, A. Science 2004, 304, 995.

(16) Nordlund, D.; Ogasawara, H.; Wernet, P.; Nyberg, M.; Odelius, M.; Pettersson, L. G. M.; Nilsson, A. Chem. Phys. Lett. 2004, 395, 161. (17) Bianconli, A. Appl. Surf. Sci. 1980, 6, 392.

(18) Romberg, R.; Frigo, S. P.; Ogurtsov, A.; Feulner, P.; Menzel, D. Surf. Sci. 2000, 451, 116.

(19) Wilson, K. R.; Rude, B. S.; Smith, J.; Cappa, C.; Co, D. T.; Schaller, R. D.; Larsson, M.; Catalano, T.; Saykally, R. J. Rev. Sci. Instrum. 2004 75,725 .

(20) Hempelmann, A.; Piancastelli, M. N.; Heiser, F.; Gessner, O.; Rudel, A.; Becker, U. J. Phys. B-At. Mol. Opt. Phys. 1999, 32, 2677.

(21) Andersen, J. N.; Johanssson, U.; Nyholm, R.; Sorenson, S. L.; Wiklund, M. MaxLab Annu. Rep. 1997, 180, 180.

(22) Mase, K.; Nagasono, M.; Tanaka, S.; Urisu, T.; Ikenaga, E.; Sekitani, T.; Tanaka, K. Surf. Sci. 1997, 390, 97.

(23) Coulman, D.; Puschmann, A.; Hofer, U.; Steinruck, H. P.; Wurth, W.; Feulner, P.; Menzel, D. J. Chem. Phys. 1990, 93, 58.

(24) Tolk, N. H. Desorption induced by electron transitions: DIET I: proceedings of the first international workshop, Williamsburg, Virginia, USA, May 12-14; Springer-Verlag: Berlin; New York, 1982.
(25) Scheuerer, R.; Feulner, P.; Wiethoff, P.; Wurth, W.; Menzel, D. J. Electron Spectrosc. Relat. Phenom. 1995, 75, 161.

(26) Sarkar, S.; Joarder, R. N. J. Chem. Phys. 1993, 99, 2032.

(27) Svishchev, I. M.; Kusalik, P. G. J. Chem. Phys. 1994, 100, 5165.

(28) Prince, K. C.; Richter, R.; de Simone, M.; Alagia, M.; Coreno, M. J. Phys. Chem. A 2003, 107, 1955.

(29) Triguero, L.; Pettersson, L. G. M.; Åren, H. Phys. Rev. B-Condens. Matter 1998, 58, 8097.

(30) Slater, J. C. Quantum Theory of Molecules and Solid; McGrawHill: 1974.

(31) Kolczewski, C.; Puttner, R.; Plashkevych, O.; Ågren, H.; Staemmler, V.; Martins, M.; Snell, G.; Schlacter, A. S.; Sant' Anna, M.; Kaindl, G.; Pettersson, L. G. M. J. Chem. Phys. 2001, 115.

(32) Casida, M. E.; Daul, C.; Goursot, A.; Hermann, K.; Koester, A.; Pettersson, L. G. M.; Proynov, E.; St-Amant, A.; Salahub, D. R.; (Principal authors); Carravetta, V.; Duarte, H.; Godbout, N.; Guan, J.; Jamorski, C.; Leboeuf, M.; Malkin, V.; Malkina, O.; Nyberg, M.; Pedocchi, L.; Sim, F.; Triguero, L.; Vela, A. (Contributing authors). deMon-KS StoBe version 1.0, deMon Software, 2002.

(33) Tsuchida, E.; Kanada, Y.; Tsukada, M. Chem. Phys. Lett. 1999. $311,236$.

(34) Franken, K. A.; Jalaie, M.; Dykstra, C. E. Chem. Phys. Lett. 1992, $198,59$.

(35) Huzinaga, S. J. J. Chem. Phys. 1965, 42, 1293.

(36) Pettersson, L. G. M.; Wahlgren, U.; Gropen, O. J. Chem. Phys. 1987, 86, 2176.

(37) Agren, H.; Carravetta, V.; Vahtras O.; Pettersson L. G. M. Theor Chem. Acc. 1997, 97, 14.

(38) Becke, A. D. Phys. Rev. A 1998, 38, 3098.

(39) Perdew, J. P. Phys. Rev. B 1986, 33, 8822.

(40) Becke, A. D. J. Chem. Phys. 1993, 98, 5648.

(41) Lee, C.; Yang, W.; Parr, R. G. Phys. Rev. B 1988, 37, 785.

(42) Frisch, M. J.; Trucks, G. W.; Schlegel, H. B.; Scuseria, G. E.; Robb, M. A.; Cheeseman, J. R.; Zakrzewski, V. G.; Montgomery, J. A., Jr.; Stratmann, R. E.; Burant, J. C.; Dapprich, S.; Millam, J. M.; Daniels, A. D.; Kudin, K. N.; Strain, M. C.; Farkas, O.; Tomasi, J.; Barone, V.; Cossi, M.; Cammi, R.; Mennucci, B.; Pomelli, C.; Adamo, C.; Clifford, S.; Ochterski, J.; Petersson, G. A.; Ayala, P. Y.; Cui, Q.; Morokuma, K.; Salvador, P.; Dannenberg, J. J.; Malick, D. K.; Rabuck, A. D.; Raghavachari, K.; Foresman, J. B.; Cioslowski, J.; Ortiz, J. V.; Baboul, A. G.; Stefanov, B. B.; Liu, G.; Liashenko, A.; Piskorz, P.; Komaromi, I.; Gomperts, R.; Martin, R. L.; Fox, D. J.; Keith, T.; Al-Laham, M. A.; Peng, C. Y.; Nanayakkara, A.; Challacombe, M.; Gill, P. M. W.; Johnson, B.; Chen, W.; Wong, M. W.; Andres, J. L.; Gonzalez, C.; Head-Gordon, M.; S., R. E.; Pople, J. A. Gaussian 98, revision A.7 ed.; Gaussian, Inc.: Pittsburgh, PA, 2001.

(43) Wight, G. R.; Brion, C. E. J. Electron Spectrosc. Relat. Phenom. 1974, 4, 25 .

(44) Sham, T. K.; Yang, B. X.; Kirz, J.; Tse, J. S. Phys. Rev. A 1989, 40,652 .

(45) Guo, J. H.; Luo, Y.; Augustsson, A.; Kashtanov, S.; Rubensson, J. E.; Shuh, D. K.; Agren, H.; Nordgren, J. Phys. Rev. Lett. 2003, 91, 157401.

(46) Wilson, K. R.; Tobin, J. G.; Ankudinov, A. L.; Rehr, J. J.; Saykally, R. J. Phys. Rev. Lett. 2000, 85, 4289. 